\title{
Lo sguardo attraverso il mito: la figura del poeta in due opere di Claudio Magris*
}

Parole chiave: letteratura italiana contemporanea, Claudio Magris, la figura del poeta, il narratore, Orfeo, Odisseo, Argonauti

DOI: 10.4312/ars.9.1.179-191

\section{Introduzione}

Entrambe le ultime opere narrative di Magris - il romanzo Alla cieca (2005) e il breve testo in prosa Lei dunque capirà (2006) - contengono una riflessione sulla figura del poeta costruita attraverso la narrazione mitologica. Mentre nel romanzo la ricerca sulla natura della voce narrante si appoggia sul mito degli Argonauti, di Odisseo e, marginalmente, su quello di Orfeo, Lei dunque capirà si incentra su quest'ultimo. Tuttavia, il cantore mitologico vi è privato della sua voce e la figura del poeta viene plasmata attraverso lo sguardo e la voce della sua compagna.

\section{La voce narrante, il mito e la cecità della storia}

In Alla cieca la storia, la sua violenza, la sua «cieca inquietudine» (Jančar, 2009, 8) si intrecciano con una riflessione sull'identità del protagonista e sulle modalità della narrazione in prima persona. L'opera è «inquadrata da un punto interrogativo» (Coda, 2012, 378): sin dall'inizio l'autore si chiede se la narrazione autodiegetica sia affatto possibile, dato che

nessuno può raccontare né conoscere sé stesso. Uno non sa com'è la sua voce; sono gli altri che la riconoscono e la distinguono. È Lei che sa quando sono io che parlo, così come io conosco Lei, voi, loro, non me. Come potrebbe Achille narrare la sua ira? (Magris, 2005a, 18) ${ }^{1}$

Ma «alle domande non c'è mai risposta» (15) e forse anche questa domanda fondamentale rimarrà sospesa nella confusione delle voci narranti che inonderanno

\footnotetext{
* La versione slovena dell'articolo è visibile sul sito http://revije.ff.uni-lj.si/arshumanitas. / Slovenska različica članka je dosegliiva na http://revije.ff.uni-lj.si/arshumanitas.

1 Per maggiore concisione le citazioni dal romanzo verranno in seguito segnate soltanto dal numero della pagina.
} 
il romanzo. È del tutto incerto «chi è che parla, di chi è quella voce» (26); le parole diventano «[r] umori, voci, un brusio, qualcosa, niente. Chi parla là dentro? [...] ascolto ma non so chi, questa voce che sale da dentro - dentro di me, [...] nessuno sa mai da dove viene una voce» (57). Il narratore autodiegetico non può definire se stesso, dal momento che «è difficile riconoscere la propria voce, non si sa come la si sente dal di fuori, come la sentono gli altri, è un'altra» (332-333). L'autore si serve delle tradizionali metafore nautiche (Curtius, 1992, 147-150) per osservare che

è solo la propria voce che si sente, come quando lassù sulla coffa si grida nel vento. Il grido si perde nel mare, quello che hai gridato lo hai sentito solo tu, ma non sei ben sicuro che sia la tua voce, forse il refolo ti ha portato quella di un altro, urlata in cima a un'altra nave sparita oltre l'orizzonte [...]; la nave fila veloce e si lascia indietro le voci salite dal ponte e dalla stiva, uccelli che volteggiano a poppa e poi restano indietro perduti. Per un po' le distingui ancora, le voci, poi è uno stridio indistinto, il vento ti sbatte in faccia e le ali degli uccelli ti stridono dentro le orecchie, voci urla parole, tutta una ciurma selvaggia e flagellata nella tua testa (15).

Rimane incerta perfino la delimitazione fra la narrazione e la lettura. Il narratore è anche il lettore, visto che «dice ora di aver letto ora di aver scritto l'autobiografia come se non si potesse scrivere e poi leggere lo stesso libro [...]. E se anche l'avessi letta prima di scriverla, non cambierebbe niente» (20; cf. anche 16, 88). In tal modo tiene contemporaneamente la posizione del narratore, del protagonista e del lettore:

ho anche mentito. Insomma, mentito - il mio autobiografo ha un po' abbellito le cose, come succede quasi sempre quando si scrive. Ho visto la mia autobiografia nel retrobottega di quella libreria antiquaria a Salamanca Place - e l'ho subito letta con passione, si capisce. Mica mi aspettavo una banale copia della realtà. Se no perché l'avrei letta, solo per trovare cose che conoscevo già? Era il resto, che mi interessava (127).

Secondo Magris l'io, sia nel senso del personaggio letterario sia nel senso del narratore autodiegetico, si sta disgregando: è «un io che quasi non c'è, che è sempre sul punto di svanire, come un po' d'acqua messa dentro un'altra acqua, ma che in qualche modo continua tuttavia ad avere una sua individualità», come sottolinea nell'articolo «Identità ovvero incertezza», pubblicato due anni prima del romanzo (Magris, 2003, 520). La problematica dell'identità è, del resto, cara allo scrittore triestino che sente fortemente «la crisi e/o liberazione dell'io, la fragilità dell'io» (Magris, 2003, 521). L'autore sostiene che «[1] a parola identità dovrebbe essere sempre declinata al plurale» e che "[l]e rappresentazioni autentiche di un'identità plurima, comunque incerta e contraddittoria, non sono mai definitorie» (Magris, 2003, 522). A proposito del personaggio, osserva che 
la nostra odissea è oggi [...] l'eterno nomadismo del personaggio musiliano che muove verso sempre nuove costellazioni e interpretazioni dell'essere, si getta e si progetta in avanti mutando la sua fisionomia e la sua natura, senza lasciar figli né eredi alle proprie spalle (Magris, 1982, 48).

Tali osservazioni sono valide anche per le numerose identità assunte dal protagonista di Alla cieca. Le più importanti sono quelle di Jorgen Jorgensen e Salvatore Cippico che, in differenti epoche storiche, percorrono il mondo attraversando «luoghi [...] tra i più oscuri della Storia moderna» (Musarra-Schröder, 2007, 123) e costituiscono una specie di «biografia sdoppiata» (Pellegrini, 2006, 35).

Jorgensen fu un navigatore e avventuriero danese storicamente esistito (17801841) che prese parte alla fondazione della prima colonia penale nell'allora Terra di Van Diemen, l'odierna Tasmania, fu in seguito imprigionato a varie riprese in Europa e, finalmente, condannato alla deportazione e ai lavori forzati. Cippico, invece, è «ovviamente un personaggio inventato, [...] uno di quegli uomini che hanno attraversato le tempeste della Storia per finire a Goli Otok» (Magris, 2012, 326). Nato in Tasmania agli albori del '900 come figlio di un immigrato italiano, viene in seguito deportato in Europa per via della sua militanza comunista. Durante la seconda guerra mondiale viene coscritto nell'esercito italiano ed inviato in Dalmazia; si associa poi alle bande partigiane jugoslave, ma viene catturato dai tedeschi e deportato a Dachau. Dopo la guerra ritorna in Jugoslavia, assieme a numerosi operai comunisti friulani, per collaborare alla costruzione del giovane stato socialista (Magris, 2005b, 137). In seguito alla rottura fra Tito e Stalin, però, è imprigionato a Goli Otok, l'Isola Calva, dove viene «[s]ottoposto [...] a lavoro bestiale e massacrante, sevizie e torture» (14), similmente a quanto accade ad altri comunisti italiani immigrati in Jugoslavia che sono rimasti fedeli a Stalin. Quando riesce finalmente a fuggire verso l'Italia condivide la sorte degli altri reduci, considerati dalla polizia dei «pericolosi comunisti provenienti dall'Est» (Magris, Badnjevic, 2008), e ignorati dal partito comunista italiano, nel frattempo riavvicinatosi a Tito, in quanto «scomodi testimoni della politica stalinista del partito che si voleva dimenticare» (Magris, Badnjevic, 2008). Pertanto, Cippico ritorna in Australia. Non è chiaro in quale luogo narri la sua storia - forse si trova in Tasmania (e narra, presumibilmente, nella propria tomba), oppure è ricoverato in una clinica psichiatrica triestina in quanto «[o]ssessionato dalla deportazione a Goli Otok a opera degli jugoslavi nel '49» (15). Sia Cippico che Jorgensen hanno «mille echi, evocano una miriade di microstorie, recuperano le voci di tanti destini minimi sciupati e cancellati dalla violenza della grande storia» (Pellegrini, 2006, 36).

Il narratore «riprende instancabilmente i fili di un discorso su se stesso, identificandosi di volta in volta con personaggi storici o con eroi mitici» (Airoldi Namer, 
2006, 267). Le identità da lui assunte si accavallano e si soppiantano senza soluzione di continuità. La voce narrante «diventa una moltitudine» (Magris, 2003, 520) dato che non appartiene più ad un solo individuo bensì coinvolge numerosi destini «macinati» dalla storia «come prede fra le zanne e nello stomaco di una bestia» (Magris, 2006b, $35)^{2}$. Pertanto, il narratore si chiede: «chi sono, chi ero, chi siamo» (12), e si definisce «io o un altro» (11) dal momento che narra «la vita, tante vite» (11). Le voci narranti sono come i televisori nella vetrina di un negozio che il protagonista guarda: da quelle «scatole luminose» escono «[t]ante voci, la stessa [...]; altre voci, la stessa voce» (316), creando «un monologo delirante nel quale le altre voci confluiscono, si intrecciano e si sovrappongono» (Magris, 2012, 329).

Il romanzo è pervaso dal mito argonautico, con ulteriori riferimenti al mito di Odisseo e, marginalmente, a quello di Orfeo. La fonte esplicita del mito argonautico è il poema di Apollonio Rodio del III secolo a. C. ${ }^{3}$, al quale si riallacciano numerosi riferimenti intertestuali nel romanzo. Ciononostante, un numero notevole di citazioni riconduce al poema bizantino Argonautiche orfiche, una tarda variante del mito risalente al V secolo d. C. ${ }^{4}$ che, però, non viene menzionata se non in un altro testo di Magris (Magris, 2012, 329). Il personaggio centrale del poema bizantino è il cantore mitologico Orfeo, al quale appartiene anche la voce narrante e che, tradizionalmente, venne considerato il suo autore leggendario. In Alla cieca la voce narrante è legata ad entrambe le fonti visto che assume, fra le altre, le identità di Apollonio Rodio e di Orfeo, e diventa «[q]uell'Apollonio [...] che pretende di narrare la storia, di essere Orfeo tra gli argonauti» (63; cf. anche 60, 133, 169).

Sia Cippico che Jorgensen si identificano con la figura mitologica di Giasone. Mentre di lui si narra, per lo più, in terza persona, in alcuni brani il narratore assume la voce, in prima persona, dell'eroe mitologico. Il seguente brano è un esempio di come la voce narrante passi direttamente da Cippico (nella prima frase) a Giasone (nella seconda): «[h]o passato tante notti insonni a Goli Otok. Sulla prua di Argo invece era così facile addormentarsi, abolire le cose» (89). Magris si chiede se la narrazione autobiografica possa essere veridica oppure se - similmente a quanto avviene per il mito argonautico - si tratti solo di un'abile «operazione di marketing» (Magris, 2012, 327; Magris, Brecelj, 2007) che falsifica la memoria personale, storica e mitologica. Giasone è, infatti, «l'eroe incerto che tace ed è come se non ci fosse, di cui si sa che è meno valoroso dei suoi Argonauti [...] ma abile nel mettere in scena l'impresa eroica, mito e réclame» (Magris, 2001, 167). Fra le caratteristiche

2 È un'espressione usata da Magris a proposito dell'Aurora boreale di Jančar.

3 La fonte usata da Magris è la traduzione italiana del poema (Apollonio Rodio, 2010); il titolo dell'opera viene esplicitato a varie riprese $(34,36,272)$. 
dell'eroe mitologico che risalgono fino al poema di Apollonio e che vengono riprese dai personaggi di Magris vi sono:

una disponibilità a tutto che giunge fino all'inconsistenza e al vuoto, una mancanza di centro e di carattere genialmente ammantata di seduzioni, un'assenza di qualità sostanziali che si presta a essere rivestita di efficacissime qualità di superficie, diplomazia, fascino, gesto eroico (Magris, 1994, 21).

Nel mito di Giasone che «porta nella Colchide morte e sventura» (288) si rispecchia la fallita impresa dei comunisti italiani che si avviano fra $\mathrm{i}$ «barbari colchici slavi, ai confini del mondo» (64) e, come gli argonauti, «spariscono nelle fauci del drago» (178) nella Jugoslavia dopo il Cominform. Jorgensen, che partecipa alla colonizzazione della Tasmania, vi «porta la luce della civiltà [...] e porta insieme nuova barbarie» (66), similmente a quanto avviene per Giasone in Colchide. Anche la letteratura fa parte di questa nuova, ambigua civiltà:

Stiamo fondando un paese [...] e dunque, come è ovvio, pure la sua letteratura. [...] Ai primordi, la letteratura è ancora intrisa di terra e di sangue; ne ha ancora l'odore addosso, cronaca di Romolo che racconta l'uccisione di Remo. Poi la carta passa di mano in mano, come il danaro, e a furia di stropicciarsi perde quell'odore, diventa sudicia ma moralmente presentabile (269-270).

Attraverso la figura di Giasone sono filtrati anche i rapporti distruttivi che il protagonista intrattiene con le donne: infatti, dopo che «una Nausicaa o una Issipile o una Medea per l'Ulisse o il Giasone di turno» (99) si sono sacrificate per lui, vengono abbandonate.

Verso la fine del romanzo le identità frammentate si fondono nella narrazione, in prima persona, della propria morte. I romanzi vengono, infatti, paragonati alle lapidi che rappresentano dei «romanzi concentrati. O meglio, i romanzi sono lapidi dilatate [...]. La mia autobiografia è una di queste lapidi dilatate» $(302)^{5}$. La narrazione della propria morte dovrebbe, finalmente, condurre il narratore alla conoscenza di se stesso:

Scrivere e mettere in scena la propria morte, come un attore che abbia studiato la sua parte. E allora saprò chi sono, perché è la morte, è il rogo, è il tumulo che narra la storia di un uomo, anche a lui stesso, meglio delle biografie e autobiografie (161).

Similmente a quanto avviene per Giasone, il protagonista muore quando gli cade addosso la polena di una nave incagliata sotto la cui prua si era sdraiato. Nella propria

5 Sembra probabile che l'autore abbia avuto l'idea di questo parallelo in occasione di una visita in Australia; nell'opera L'infinito viaggiare, pubblicata nello stesso anno di Alla cieca, commenta così la sua visita nell'ex colonia penale di Port Arthur: «Sulla vicina 'isola dei morti' ci sono le tombe dei detenuti; le lapidi sono romanzi condensati di vite incredibili» (Magris, 2005b, 243). 
narrazione si riallaccia al parallelo fra lapidi - «voglio ben credere che abbiano curato la mia lapide come si deve» (321) - e l'autobiografia, nella quale «è logico che tutto questo non ci sia e ancor meno nelle biografie. Solo io, per forza di cose, posso sapere la verità dell'Ultimo Giorno [...] cosa è successo e come» (321). La voce narrante in prima persona si fonde finalmente con quella di Giasone (331-332, 334-335):

Argo, da quando l'avevo consacrata a Poseidone e lasciata in riva al mare, marciva sì e andava in pezzi, ma i devoti che venivano a venerarla li cambiavano continuamente, sostituivano ora l'uno ora l'altro e così la nave è sempre rimasta là, antica e nuova, intatta e immortale, un'altra e la stessa, come me, come gli dèi (331).

Con il racconto della propria morte si conclude anche la riflessione sulla narrazione autodiegetica. Il dubbio iniziale sulla fattibilità di tale narrazione - «non sono sicuro,

anche se sono stato io a scriverlo, che nessuno possa raccontare la vita di un uomo meglio di lui stesso» (9) - trova, infine, una risposta nello sguardo dell'altro:

La mia vita è quella che mi raccontano gli altri. [...] Questo non vale solo per la prima infanzia. Vale per ogni istante della vita. [...] non mi sono visto, non mi conosco. Ma se Lei me lo dice, lo so e posso raccontarlo (176).

\section{La figura del poeta attraverso lo sguardo della musa}

Il breve testo in prosa Lei dunque capirà è «una rivisitazione moderna del mito di Orfeo ed Euridice» in cui, però, «la parola viene data alla donna, a Euridice» (Magris, 2008, 28). Il testo esplora $\mathrm{i}$ «sentimenti di dolore e di impotenza che accompagnano la morte di una persona cara» (Calvo Revilla, 2010, 139) e, allo stesso tempo, riflette sulla figura del poeta. La voce narrante in questo «monologo al femminile» (Magris, 2008, 28) può essere attribuita a Euridice, ad una qualsiasi donna morta oppure ad un'ospite di una casa di riposo. In questo tipo di narrazione, A. Milano Appel riconosce delle «immagini speculari», e spiega: «vi è un lui (Magris autore) che scrive con la voce di una lei (la narratrice Euridice) che parla di un altro lui (suo marito, il poeta Orfeo) e la loro vita insieme. Così abbiamo un lui, Orfeo, visto da una lei, Euridice, vista da lui autore» (Milano Appel, 2011, 38). Benché la figura della protagonista tragga le sue origini dalla cultura greca da cui «nascono le più grandi figure femminili della letteratura mondiale, che incarnano l'assoluto [...] e che per secoli, millenni, nutriranno la creatività occidentale, che sempre ritornerà ad esse per dar voce e figura all'universaleumano» (Magris, Rubino, 2011), il testo di Magris effettua una banalizzazione della storia mitologica nonché della figura del poeta.

La voce narrante femminile si fa sentire da una vaga "Casa di riposo» che si trova «dall'altra parte» (Magris, 2006a, 54) e in cui regna il buio: «qui dentro si vede 
poco, un'ombra scivola via prima che la si possa guardare in viso, a parte che tutti si assomigliano, ci assomigliamo, è logico, in una Casa come questa» (Magris, 2006a, 9). Per l'autore, la misteriosa casa è «probabilmente simbolo della vita dopo la morte, di ciò che sta oltre la morte» (Magris, 2008, 28), e la protagonista vi si ritrova «fra tutti quegli altri, incartapecoriti come mummie, sporchi» (Magris, 2006a, 12). Le severe regole della Casa

vietano agli ospiti - nel loro, nel nostro interesse - di ricevere visite e di mettere a repentaglio la propria pace e la propria tranquillità, figuriamoci poi di uscire, si capisce, ci mancherebbe, trovarsi in quella bolgia, in quel caos di traffico e di gente maleducata o peggio, per non parlare del tempaccio, da cui qui almeno siamo al riparo (Magris, 2006a, 10).

La tenebrosa e tranquilla Casa di riposo con i suoi ospiti che sembrano delle mummie oscilla, dunque, fra una dimensione mitologica dell'aldilà ed una percezione del tutto quotidiana, dal momento che viene contrapposta a faccende banali quali un denso traffico e il maltempo.

Nel suo monologo, la narratrice si rivolge al cosiddetto «signor Presidente», secondo l'autore «indubbiamente un'allusione a Dio» (Magris, 2008, 28), che è «invisibile, ma sembra essere il grande costruttore e narratore, a volte incomprensibile» (Magris, 2008, 29). Il personaggio maschile del quale si narra, in parte, alla terza persona e, in parte, alla seconda, viene definito come poeta o autore:

Se pensate che esageri, diceva agli amici, vuol dire che non avete cuore né sentimento, non avete poesia nel cuore, chi potrà giammai capire la mia pena e il mio tormento, la sofferenza, il dolore di un poeta... (Magris, 2006a, 13).

La protagonista è la sua musa e «ad una Musa si obbedisce, no?» (Magris, 2006a, 15). La musa controlla, razionalizza e pone dei limiti alla sua creazione, banalizzandola attraverso metafore che richiamano faccende domestiche. Il mito di Orfeo viene così attualizzato e, allo stesso tempo, banalizzato:

Io gliele sforbiciavo, certo, le sue parole - lui, eccessivo e smodato e magnanimo, com'è sempre stato, profondeva parole a piene mani e io gliele sbucciavo, buttavo via la scorza, il torsolo e anche tanta polpa, quando era necessario. Lui non ne sarebbe stato capace, avido e incontinente e compulsivo com'era, sempre un boccone e un bicchiere di troppo, ma da me si lasciava mettere a dieta e sapeva che, se restava qualcosa sul piatto dopo che avevo passato tutto al setaccio, era veramente qualcosa di buono (Magris, 2006a, 14).

Se il poeta ripete fedelmente quanto gli viene dettato dalla musa, guadagna il lauro dei premi letterari che "porta a casa e la sua Musa glielo mette nell'arrosto che gli prepara con tanto amore, perché così viene più saporito» (Magris, 2006a, 15). Per via 
di tali banalizzazioni la voce narrante «è spogliata delle sue illusioni e può, a volte, sembrare quasi cinica» (Milano Appel, 2011, 45).

La protagonista viene ricoverata nella Casa di riposo per un'indefinita grave malattia: «Dio sa se non stavo male, con quella maledetta infezione, neanche mi avesse morsa un serpente velenoso» (Magris, 2006a, 12), il che è una chiara allusione alla morte di Euridice (Virgilio, Georgiche, IV, 457-459; Ovidio, Metamorfosi, X, 8-10). Il suo compagno si dispera finché ottiene il permesso di entrare nella Casa e portarla via $^{6}$; questo, però, viene realizzato «non grazie alla forza della sua poesia, bensì ottemperando a [...] procedure burocratiche» (Parmegiani, 2011, 125). Tuttavia, prima che possano uscire dalla Casa, il poeta si volge per guardarla, similmente a quanto avviene per Orfeo prima di poter ricondurre Euridice alla luce del sole (Virgilio, Georgiche, IV, 485-506; Ovidio, Metamorfosi, X, 53-63). Ma nell'opera di Magris

non è come hanno detto, che si è girato per troppo amore, incapace di pazienza e di attesa, e dunque per troppo poco amore. E nemmeno perché, se fossi tornata con lui, da lui, non avrebbe più potuto cantare quelle canzoni melodiose e struggenti che dicevano il dolore della mia perdita e di ogni perdita e avevano fatto il giro del mondo, diffuse dai juke box e poi dai cd, amate da tutti, che avrebbero continuato ad amarle solo se le avesse cantate ancora e ne avesse cantate altre come quelle, lo strazio per la mia lontananza, il vento che muoveva le corde della sua lira, che lo faceva poeta solo se era senza di me per la pena di essere senza di me (Magris, 2006a, 52-53).

L'essenza della poesia è «[c]antare il segreto della vita e della morte [...], chi siamo donde veniamo dove andiamo, ma duro è il confine, la penna si spezza contro le porte di bronzo che nascondono il destino» mentre, per il poeta, «soltanto il Vero grande e terribile è degno del canto [...] e quel Vero lo si conosce soltanto dietro le porte» (Magris, 2006a, 47-48). Magris spiega che la stesura del monologo è stata influenzata in modo cruciale da un'«esperienza della soglia» (Magris, 2008, 28). Per vari anni aveva, infatti, visitato una donna anziana in una casa di riposo triestina. Ogni volta che ne attraversava la soglia si ritrovava «in un mondo totalmente diverso» (Magris, 2008, 28), motivo per cui si chiedeva se capisse meglio il mondo dentro o fuori, «davanti allo specchio o dietro ad esso» (Magris, 2008, 28).

Nella risemantizzazione del mito in Lei dunque capirà il poeta si gira perché così vuole la protagonista; «[1] ui voleva sapere e io gliel'ho impedito» (Magris, 2006a, 54). Infatti,

[1]à fuori [...] si smania di sapere [...]. Lui poi smania più di tutti, perché è un poeta e la poesia, dice, deve scoprire e dire il segreto della vita, strappare il velo, sfondare le porte, toccare il fondo del mare dov'è nascosta la perla. Forse, 
ho pensato, era venuto a prendermi soprattutto - soltanto? - per questo, per sapere, per interrogarmi, perché gli raccontassi ciò che sta dietro queste porte e lui potesse afferrare la sua lira e inalzare [sic] il canto nuovo, inaudito, il canto che dice ciò che nessuno sa (Magris, 2006a, 48).

La protagonista si chiede: «[c] ome dirgli che, qui dentro, a parte la luce tanto più fioca, è come là fuori? Che siamo dietro lo specchio, ma che quel retro è anch'esso uno specchio, uguale all'altro» (Magris, 2006a, 49). Pertanto, la protagonista decide di risparmiargli «la terribile scoperta che perfino nell'aldilà [...] noi non capiamo molto di più di quanto capiamo qui, in vita» (Magris, 2008, 28):

Lei dunque capirà, signor Presidente, perché, quando eravamo ormai prossimi alle porte, l'ho chiamato con voce forte e sicura, la voce di quando ero giovane, dall'altra parte, e lui - sapevo che non avrebbe resistito - si è voltato, mentre io mi sentivo risucchiare indietro, leggera, sempre più leggera, una figurina di carta nel vento, un'ombra che si allunga si ritira e si confonde con le altre ombre della sera, e lui mi guardava impietrito ma saldo e sicuro e io svanivo felice al suo sguardo, perché già lo vedevo ritornare straziato ma forte alla vita, ignaro del nulla, ancora capace di serenità, forse anche di felicità (Magris, 2006a, 54-55).

Così il mito «dichiara il suo scacco, l'impotenza dell'uomo e del poeta, la sua definitiva rassegnazione» (De Michelis, 2010, 447). Attraverso lo sguardo di Euridice viene presentato un Orfeo «poeta di carne e ossa, disorientato, perplesso, strano, timido e pauroso, solitario e malinconico, tormentato da scrupoli di coscienza e rimorsi, nevrotico e confuso» (Calvo Revilla, 2010, 146). Nonostante la banalizzazione del mito e della figura del poeta, però, lo sguardo femminile è essenziale per l'identità di quest'ultimo: «[c]on te, diceva, vicino a te so chi sono e non sono niente male» (Magris, 2006a, 14). D'altronde, anche la musa si rispecchia nello sguardo del poeta (Milano Appel, 2011, 46):

Quando era ormai chiaro che stavo per trasferirmi nella Casa e tu passavi le ore al mio letto, mi vedevo così bella, nei tuoi occhi; mi desideravo attraverso il tuo sguardo; sapevo di essere bianca e pallida, spossata da quel veleno, ma nei tuoi occhi ero ancora bruna di sole e di mare come quando andavamo su quella nostra piccola isola, la raggiungevamo a nuoto e sbarcavamo fra lo stridere dei gabbiani, nudi e splendenti come dèi (Magris, 2006a, 32).

Lo sguardo serve da tramite anche nell'espressione del «desiderio del poetamarito di conoscere il mistero dell'aldilà - attraverso Euridice, riportata fra i viventi e di poterlo riferire agli uomini nella sua poesia» (Parmegiani, 2011, 127). Lo sguardo si rispecchia in un altro sguardo, ma non può attraversare la soglia della conoscenza e penetrare dietro l'immagine rispecchiata. 
In entrambe le opere di Magris il mito è uno degli elementi chiave che orientano la riflessione su alcune fondamentali questioni metaletterarie. «Il mito e lo sguardo» diventa così «lo sguardo attraverso il mito» e, allo stesso tempo, il rispecchiarsi dello sguardo, la formazione dell'identità attraverso la risemantizzazione del mito e attraverso lo sguardo dell'altro.

\section{Bibliografia}

Airoldi Namer, F., Straniero da dove? Claudio Magris e il romanzo «Alla cieca», Narrativa 28, 2006, pp. 267-280.

Apollonio Rodio, Le argonautiche (trad. Paduano, G., ed. Paduano, G., Fusillo, M.), Milano 2010.

Argonautiche orfiche (ed. Migotto, L.), Pordenone 1994.

Calvo Revilla, A., Escribir la ausencia, recordando a Orfeo: Lei dunque capirà de Claudio Magris, Cuadernos de Filología Italiana 17, 2010, pp. 139-152.

Coda, E., Utopia and disenchantment in Claudio Magris's Alla cieca, Journal of European Studies 42, 2012 (4), pp. 375-389.

Curtius, E. R., Letteratura europea e Medio Evo latino (trad. Luzzatto, A., Candela, M., Bologna, C.), Firenze 1992.

De Michelis, C., In viaggio con Claudio Magris, in: Moderno antimoderno: studi novecenteschi, Torino 2010, pp. 415-447.

Jančar, D., La Storia, la sua cieca inquietudine, in: Incontro Claudio Magris - Drago Jančar in occasione della traduzione in sloveno dei libri Lei dunque capirà e Alla cieca, Lubiana, Cankarjev dom, 26 novembre 2009 (ed. Ferrazza, R., Zecca, M., trad. Brecelj, V.), pp. 8-11.

Magris, C., Itaca e oltre, in: Itaca e oltre: i luoghi del ritorno e della fuga in un viaggio attraverso alcuni grandi temi della nostra cultura, Milano 1982, pp. 46-47.

Magris, C., Medea. Tragedia dello straniero, Corriere della Sera, 17 aprile 1994, p. 21, http://archiviostorico.corriere.it/1994/aprile/17/Medea_tragedia_dello_straniero_ co_0_940417176.shtml [25.6.2012].

Magris, C., Assirtidi, in: Microcosmi, Milano 2001, pp. 151-188.

Magris, C., Identità ovvero incertezza, Lettere italiane 4, 2003, pp. 519-527.

Magris, C., Alla cieca, Milano 2005a.

Magris, C., L'infinito viaggiare, Milano 2005b.

Magris, C., Lei dunque capirà, Milano 2006a. 
Magris, C., Mitteleuropa: Se i demoni del mito rivivono nell'odio tra le nazioni, Corriere della Sera, 22 agosto 2006b, p. 35, http://archiviostorico.corriere.it/2006/ agosto/22/Mitteleuropa_demoni_del_mito_rivivono_co_9_060822019.shtml [25. 6. 2012].

Magris, C., Brecelj, V., Svet se bo podrl kot peščeni grad [Il mondo crollerà come un castello di sabbia], Dnevnik, 24 febbraio 2007, pp. 22-23, http://narocanje.dnevnik. si/objektiv/230544 [25. 6. 2012].

Magris, C., The Self That Writes, discorso pronunciato in occasione dell'evento letterario Almost Island Dialogues: Two, New Delhi 2008a, http://almostisland. com/monsoon_2008/essay/pdfs/the_self_that_writes.pdf [24. 6. 2012].

Magris, C., Badnjevic, D., Gli eroi sbagliati dell'Isola Nuda, Corriere della Sera, 8 novembre 2008b, http://www.corriere.it/cultura/08_novembre_08/isola_ nuda_0610e0de-ad6b-11dd-8ab4-00144f02aabc.shtml [24. 6. 2012].

Magris, C., Rubino, M., L'era dell'emancipazione è rimasta senza eroine, Corriere della Sera, 14 novembre 2011, http://www.corriere.it/cultura/11_novembre_14/magris_ emancipazione_18d5fe98-0eb6-11e1-98bb-351bac11bfea.shtml [24. 6. 2012].

Magris, C., Narrating history, inventing history: the making of Blindly, Journal of European Studies 42 (4), 2012, pp. 324-332.

Milano Appel, A., Mirror Images of Remembrance in Marisa Madieri's La conchiglia and Claudio Magris's Lei dunque capirà: A Translator's Notes, Quaderni d'italianistica XXXII/1, 2011, pp. 37-47.

Musarra-Schröder, U., La geografia della Storia: «Alla cieca» di Claudio Magris, Otto / Novecento 1, 2007, pp. 123-135.

Ovidio, Metamorfosi (ed. Scivoletto, N.), Torino 2005.

Parmegiani, S., The Presence of Myth in Claudio Magris's Postmillennial Narrative, Quaderni d'italianistica XXXII/1, 2011, pp. 111-134.

Pellegrini, E., La biografia sdoppiata di Magris: «Alla cieca» di Claudio Magris, La rivista dei libri - The New York Review of Books 4, 2006, pp. 35-38.

Prosenc, I., Mit o Argonavtih v romanu Na slepo Claudia Magrisa [Il mito degli Argonauti in Alla cieca di Claudio Magris], Keria 14/2, 2012, pp. 59-71.

Prosenc, I., "Salire, no, discendere agli dèi": la navigazione mitologica in Alla cieca di Claudio Magris, Gaia (Université de Grenoble) 15, 2012, pp. 225-252.

Prosenc, I., "Zgodovina ne teče naravnost, cikcakasto gre": percepcija časa v Magrisovem romanu $\mathrm{Na}$ slepo [La storia non è lineare [...], va a zig-zag: la percezione del tempo nel romanzo Alla cieca di Claudio Magris], Ars \& Humanitas VI/2, 2012, pp. 9-22.

Publio Virgilio Marone, Georgiche (ed. Ramous, M.), Milano 2009. 
Irena Prosenc

\section{Pogled skozi mit: lik pesnika v dveh delih Claudia Magrisa}

Ključne besede: sodobna italijanska književnost, Claudio Magris, lik pesnika, pripovedovalec, Orfej, Odisej, Argonavti

Prispevek obravnava lik pesnika, izoblikovan prek pogleda drugega in hkrati prek prizme mitološke pripovedi, $\mathrm{v}$ dveh delih Claudia Magrisa: kratkem proznem delu Saj razumete (Lei dunque capirà) in romanu Na slepo (Alla cieca). V prvem besedilu podobo pesnika izoblikuje Evridikin glas. Besedilo prek Evridikinega pogleda zre na mit o Orfeju, ga aktualizira in morda tudi banalizira. Roman $\mathrm{Na}$ slepo prek mita o Argonavtih, prepletenega z Odisejevim in Orfejevim mitom, razmišlja o tem, komu pripada pripovedni glas, ali je avtobiografska pripoved sploh lahko verodostojna ali pa je - hkrati z mitološko pripovedjo - nemara le »réclame« in torej predstavlja izkrivljen pogled na osebni, zgodovinski in mitološki spomin. Magris se hkrati sprašuje, ali v sodobni književnosti še obstaja trden pripovedni glas ali pa je ta razdrobljen in negotov glede lastne identitete. V obeh delih je mit torej osrednji element, ki omogoči vpogled v nekatera pomembna metaliterarna vprašanja. »Mit in pogled « tako postane »pogled skozi mit« in hkrati zrcaljenje pogleda: »Nisem se videl, ne poznam se. Če pa mi vi to poveste, vem in lahko pripovedujem.« 
Irena Prosenc

\section{Looking through the Myth: the Figure of the Poet in Two Works by Claudio Magris}

Keywords: contemporary Italian literature, Claudio Magris, figure of the poet, narrator, Orpheus, Odysseus, Argonauts

The paper analyses the figure of the poet in two works by the contemporary Italian writer Claudio Magris: in the short prose text Lei dunque capirà (You Will Therefore Understand) and the novel Alla cieca (Blindly). In both works the figure of the poet is constructed through the eyes of the other as well as through the prism of mythological narration. In Lei dunque capirà, the image of the poet is shaped by Eurydice's voice, which filters the Orpheus myth, actualising and perhaps banalizing it. The novel, on the other hand, uses the Argonauts myth, intertwined with the Odysseus and Orpheus myths, to reflect on the nature of the narrating voice. The novel questions whether autobiographical narration can at all be reliable or whether it is to be considered along with mythological narration - as nothing more than "réclame", representing a deformed view of personal, historical and mythological memory. Magris questions the existence of a stable narrative voice in contemporary fiction, since it appears fragmented and unsure about its own identity. Both works include myth as the central element facilitating reflection on fundamental metaliterary issues. "Looking at the Myth" thus becomes "looking through the myth", as well as, in Eurydice's narration, looking at a reflection: "I haven't seen myself, I do not know myself. But if you tell me about this, I shall know it and will be able to narrate it". 